\title{
FRONTIERS OF STATISTICS
}




\section{FRONTIERS OF STATISTICS}

The book series provides an overview on the new developments in the frontiers of statistics. It aims at promoting statistical research that has high societal impacts and offers a concise overview on the recent developments on a topic in the frontiers of statistics. The books in the series intend to give graduate students and new researchers an idea where the frontiers of statistics are, to learn common techniques in use so that they can advance the fields via developing new techniques and new results. It is also intended to provide extensive references so that researchers can follow the threads to learn more comprehensively what the literature is, to conduct their own research, and to cruise and lead the tidal waves on the frontiers of statistics.

\section{SERIES EDITORS}

\author{
Jianqing Fan \\ Frederick L. Moore '18 Professor of Finance, \\ Director of Committee of Statistical Studies, \\ Department of Operation Research and \\ Financial Engineering, \\ Princeton University, NJ 08544, USA.
}

\author{
Zhiming Ma \\ Academy of Math and Systems Science, \\ Institute of Applied Mathematics, \\ Chinese Academy of Science, \\ No.55, Zhong-guan-cun East Road, \\ Beijing 100190, China.
}

\section{EDITORIAL BOARD}

T. Tony Cai, University of Pennsylvania

Min Chen, Chinese Academy of Science

Xiaotong Shen, University of Minnesota

Zhi Geng, Peking University

Xuming He, University of Illinois at Urbana-Champaign

Xihong Lin, Harvard University

Jun Liu, Harvard University

Xiao-li Meng, Harvard University

Jeff Wu, Georgia Institute of Technology

Heping Zhang, Yale University 


\section{High-Dimensional Data Analysis}

\section{Editors}

\section{T. Tony Cai}

University of Pennsylvania, USA

\section{Xiaotong Shen}

University of Minnesota, USA

\section{Volume 2}

\section{Higher Education Press}




\section{T. Tony Cai}

Department of Statistics,

University of Pennsylvania,

Philadelphia, PA 19104, USA.

\section{Xiaotong Shen}

School of Statistics, University of Minnesota, Minneapolis, MN 55455, USA.

\author{
Copyright (c) 2011 by \\ Higher Education Press \\ 4 Dewai Dajie, 100120, Beijing, P.R. China and \\ World Scientific Publishing Co Pte Ltd \\ 5 Toh Tuch Link, Singapore 596224
}

All rights reserved. No part of this book may be reproduced or transmitted in any form or by any means, electronic or mechanical, including photocopying, recording or by any information storage and retrieval system, without permission in writing from the Publisher.

\author{
ISBN 13: 978-981-4324-85-4 \\ ISBN 10: 981-283-4324-85-x \\ ISSN 1793-8155
}

Printed in P. R. China 


\section{Preface}

Over the last few years, significant developments have been taking place in highdimensional data analysis, which are driven primarily by a wide range of applications in many fields, such as genomics and signal processing. In particular, substantial advances have been made in the areas of feature selection, covariance estimation, classification and regression. This book intends to examine important issues arising from high-dimensional data analysis to explore key ideas for statistical inference and prediction. The book is structured around topics on multiple hypothesis testing, feature selection, regression, classification, dimension reduction, as well as applications in survival analysis and in biomedical research.

Fundamental statistical issues underlying data have changed, when moving from low-dimensional to high-dimensional analyses. For instance, certain structures such as sparsity need to be utilized in feature selection when the number of candidate features greatly exceeds that of the sample size. As a result of high-dimensionality, traditional statistical methods designed for low dimensional problems become inadequate or break down. To meet these challenges in highdimensional analysis, statisticians have been developing new methods and introducing new concepts, where many issues emerge with regard to how to identify or utilize certain structures for dimension reduction in inference and prediction.

There exists a vast body of literature on high-dimensional analysis, especially for prediction, classification and regression. We do not intend to give an overview of each subject but would like to mention here only a few topics of interest-feature selection, basis/grouping pursuit, multiple hypothesis testing, effective dimension reduction and projection pursuit, sparsity, high-dimensional regression and classification.

Many classification problems are often high-dimensional. In Chapter 1, Fan, Fan and Wu review contemporary classification methods, including linear discriminant analysis, naive Bayes, and loss-based methods, as well as the impact of dimensionality on classification, with a special attention towards regularization and feature selection. In Chapter 2, Liu and Wu move further to the topic of large margin classification, where they examine various state-of-art methods for various of support vector machines, and their connection with probability estimation.

In Chapter 3, Cai and Sun consider large-scale multiple testing. They begin by reviewing methods for controlling family-wise error rates and false discovery rates (FDR), as well as other pertinent issues in multiple testing. Their main focus is on optimal multiple testing procedures minimizing the false nondiscovery rate while controls the FDR. Both independent and dependent cases are considered.

The topic of high-dimensional feature (variable) selection has been a focus in recent research. In Chapter 4, Yuan reviews several popular variable selection 
methods, and contrast classical methods such as stepwise selection with modern methods such as regularization. In Chapter 5, Zhu, Pan and Shen examine Bayesian model selection for networks, particularly gene networks where the number of genes in a network may greatly exceed the sample size.

In Chapter 6, Li describes a number of interesting applications in genomics studies involving networks and graphical models, where the dimension under consideration is ultra-high. Various regression techniques have been reviewed, where special structures of genomic data are considered.

Survival data analysis is an important subject in biostatistics. Analysis highdimensional survival data requires power tools. In Chapter 7, Li and Ren focus on joint modeling for censored and longitudinal data. Various models are reviewed, subject to different types of censoring. In Chapter 8 , Nan reviews the recent development of feature selection in penalized regression in survival analysis, which is a marriage between high-dimensional feature selection and survival analysis. Several methods are examined, particularly for high-dimensional covariates such as gene expressions, whereas various penalties such as grouped, hierarchical penalties are discussed.

For high-dimensional data analysis, dimension reduction is essential. In Chapter 9, Yin gives a comprehensive review on sufficient dimension reduction in regression. In Chapter 10, Chen and Yang discuss combining strategies.

Finally, we sincerely hope that this book can simulate further interest from statisticians, computer scientists and engineers, and promote further collaborations among them to attack important problems in high-dimensional data analysis.

T Tony Cai, Philadelphia Xiaotong Shen, Minneapolis

May 18, 2010 


\section{Contents}

\section{Preface}

\section{Part I High-Dimensional Classification}

\section{Chapter 1 High-Dimensional Classification}

Jianqing Fan, Yingying Fan and Yichao $W u \ldots \ldots \ldots \ldots \ldots \ldots \ldots, 3$

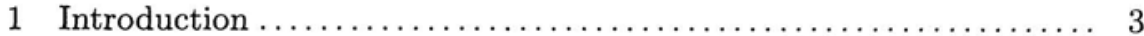

2 Elements of classifications .............................. 4

3 Impact of dimensionality on classification .................... 8

4 Distance-based classification Rules ........................ 14

5 Feature selection by independence rule $\ldots \ldots \ldots \ldots \ldots \ldots \ldots \ldots, 20$

6 Loss-based classification ................................ 24

7 Feature selection in loss-based classification $\ldots \ldots \ldots \ldots \ldots \ldots \ldots, 27$

8 Multi-category classification $\ldots \ldots \ldots \ldots \ldots \ldots \ldots \ldots \ldots \ldots \ldots \ldots \ldots \ldots \ldots \ldots$

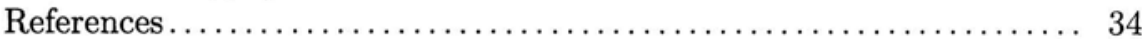

Chapter 2 Flexible Large Margin Classifiers

Yufeng Liu and Yichao $\mathrm{Wu} \ldots \ldots \ldots \ldots \ldots \ldots \ldots \ldots \ldots \ldots \ldots \ldots, \ldots \ldots \ldots$

1 Background on classification .......................... 39

2 The support vector machine: the margin formulation and the SV interpretation $\ldots \ldots \ldots \ldots \ldots \ldots \ldots \ldots \ldots \ldots \ldots \ldots, 40$

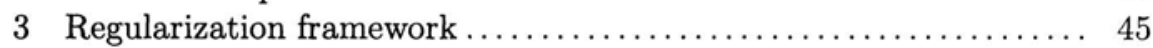

4 Some extensions of the SVM: Bounded constraint machine

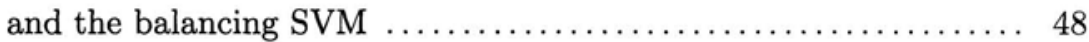

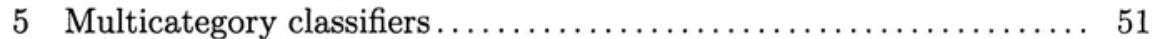

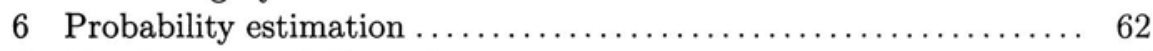

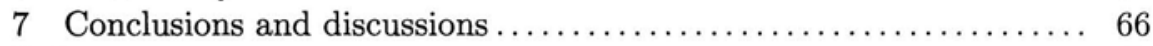

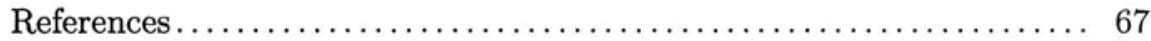

\section{Part II Large-Scale Multiple Testing}

\section{Chapter 3 A Compound Decision-Theoretic Approach to} Large-Scale Multiple Testing

T. Tony Cai and Wenguang Sun ......................... 75

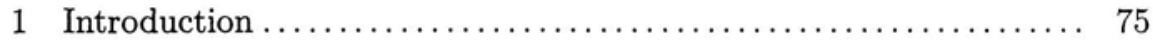

2 FDR controlling procedures based on $p$-values $\ldots \ldots \ldots \ldots \ldots \ldots, 79$

3 Oracle and adaptive compound decision rules for FDR control .... 82 
4 Simultaneous testing of grouped hypotheses $\ldots \ldots \ldots \ldots \ldots \ldots \ldots . \ldots 3$

5 Large-scale multiple testing under dependence................ 102

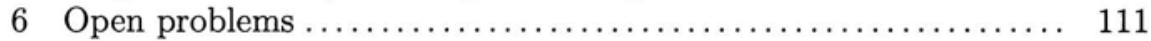

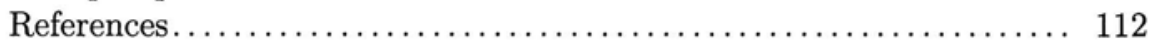

\section{Part III Model Building with Variable Selection}

\section{Chapter 4 Model Building with Variable Selection}

Ming Yuan ..................................... 119

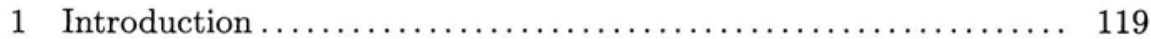

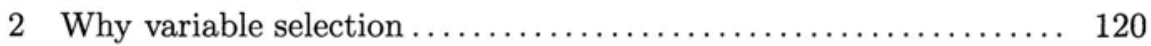

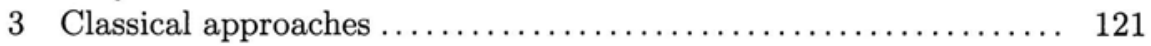

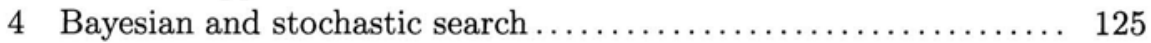

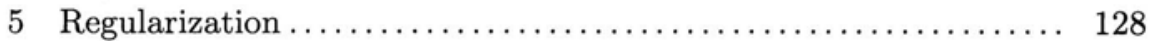

6 Towards more interpretable models ..................... 134

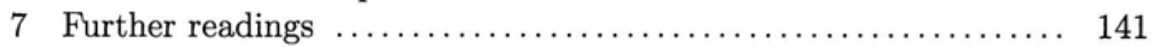

References........................................ 142

\section{Chapter 5 Bayesian Variable Selection in Regression} with Networked Predictors

Feng Tai, Wei Pan and Xiaotong Shen..................... 147

1 Introduction ....................................... 147

2 Statistical models ..................................... 149

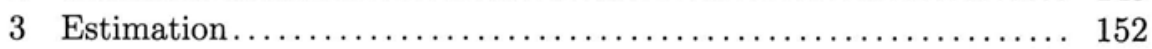

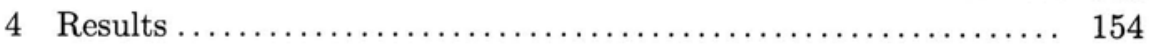

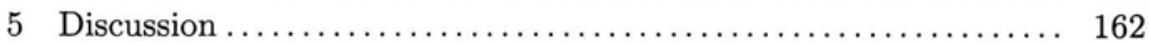

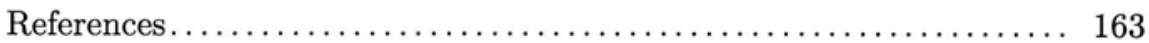

\section{Part IV High-Dimensional Statistics in Genomics}

Chapter 6 High-Dimensional Statistics in Genomics

Hongzhe Li................................... 169

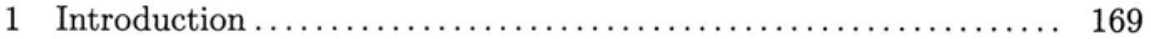

2 Identification of active transcription factors using time-course gene expression data $\ldots \ldots \ldots \ldots \ldots \ldots \ldots \ldots \ldots \ldots \ldots \ldots \ldots$

3 Methods for analysis of genomic data with a graphical structure.. 178

4 Statistical methods in eQTL studies..................... 182

5 Discussion and future direction $\ldots \ldots \ldots \ldots \ldots \ldots \ldots \ldots \ldots \ldots \ldots \ldots \ldots \ldots$

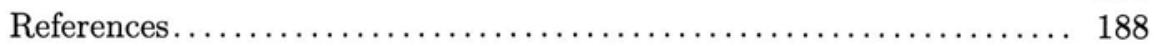

Chapter 7 An Overview on Joint Modeling of Censored Survival Time and Longitudinal Data

Runze Li and Jian-Jian Ren............................ 195

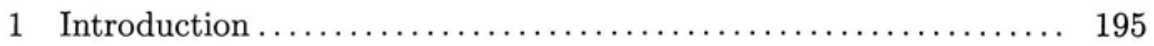


2 Survival data with longitudinal covariates ................ 197

3 Joint modeling with right censored data .................. 202

4 Joint modeling with interval censored data............... 214

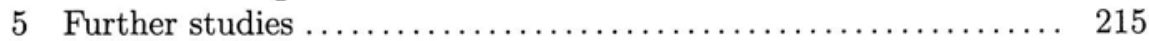

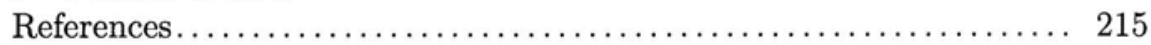

\section{Part V Analysis of Survival and Longitudinal Data}

\section{Chapter 8 Survival Analysis with High-Dimensional Covariates}

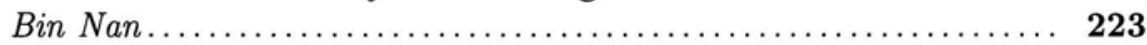

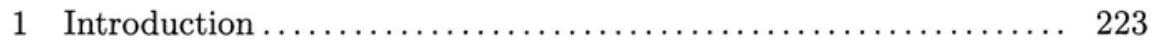

2 Regularized Cox regression ......................... 227

3 Hierarchically penalized Cox regression with grouped variables ... 232

4 Regularized methods for the accelerated failure time model...... 247

5 Tuning parameter selection and a concluding remark ......... 251

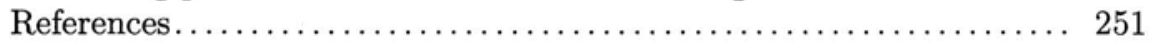

\section{Part VI Sufficient Dimension Reduction in} Regression

\section{Chapter 9 Sufficient Dimension Reduction in Regression}

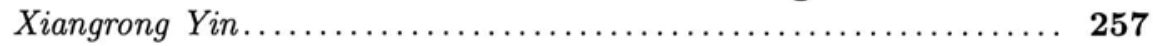

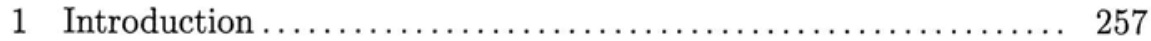

2 Sufficient dimension reduction in regression $\ldots \ldots \ldots \ldots \ldots \ldots \ldots 258$

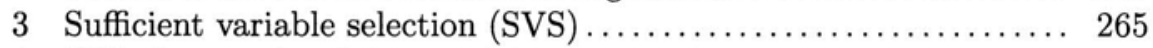

4 SDR for correlated data and large- $p$-small- $n \ldots \ldots \ldots \ldots \ldots \ldots \ldots 266$

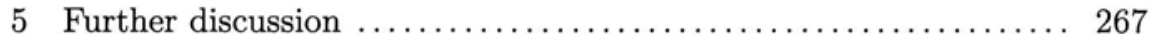

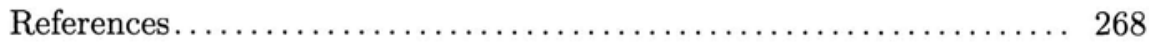

\section{Chapter 10 Combining Statistical Procedures}

Lihua Chen and Yuhong Yang .......................... 275

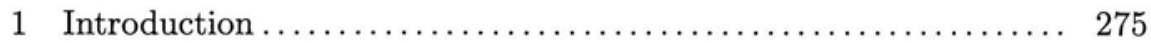

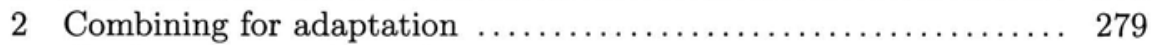

3 Combining procedures for improvement $\ldots \ldots \ldots \ldots \ldots \ldots \ldots \ldots 288$

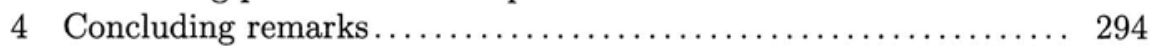

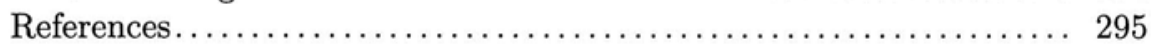

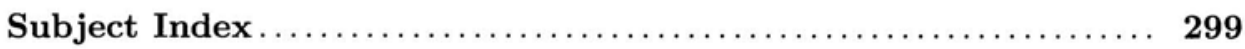

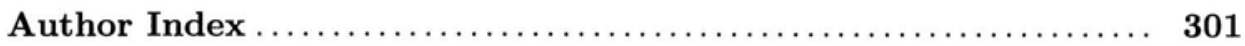

